\title{
IdeAs
}

Idées d'Amériques

$11 \mid 2018$

Modernités dans les Amériques : des avant-gardes à aujourd'hui

\section{Omar Valerio-Jiménez, Santiago Vaquera-Vásquez, Claire F. Fox (éditeurs), The Latina/o Midwest Reader} University of Illinois Press, 2017, 332 pages

\section{James Cohen}

\section{OpenEdition \\ Journals}

Édition électronique

URL : https://journals.openedition.org/ideas/2304

DOI : $10.4000 /$ ideas.2304

ISSN : $1950-570$

Éditeur

Institut des Amériques

\section{Référence électronique}

James Cohen, « Omar Valerio-Jiménez, Santiago Vaquera-Vásquez, Claire F. Fox (éditeurs), The Latina/ o Midwest Reader », IdeAs [En ligne], 11 | 2018, mis en ligne le 18 mai 2018, consulté le 19 octobre 2022. URL : http://journals.openedition.org/ideas/2304 ; DOI : https://doi.org/10.4000/ideas.2304

Ce document a été généré automatiquement le 19 octobre 2022.

\section{(c) (i) $\odot$}

Creative Commons - Attribution - Pas d'Utilisation Commerciale - Pas de Modification 4.0 International - CC BY-NC-ND 4.0

https://creativecommons.org/licenses/by-nc-nd/4.0/ 


\section{Omar Valerio-Jiménez, Santiago Vaquera-Vásquez, Claire F. Fox (éditeurs), The Latina/o Midwest Reader}

University of Illinois Press, 2017, 332 pages

James Cohen

\section{RÉFÉRENCE}

Omar Valerio-Jiménez, Santiago Vaquera-Vásquez, Claire F. Fox (éditeurs), The Latina/o Midwest Reader, University of Illinois Press, 2017, 332 pages

1 Jusqu'au milieu des années 1990 la géographie de l'implantation des Latinos (ou Hispaniques) aux Etats-Unis était relativement simple: les Mexicains et Mexicains Américains vivaient dans le Sud-Ouest, les Portoricains dans le Nord-Est (essentiellement à New York) et les Cubains, pour l'essentiel, dans le sud de la Floride. Seule la ville de Chicago présentait l'originalité d'être historiquement un espace de rencontre entre Mexicains et Portoricains. Aujourd'hui, le tableau est infiniment plus complexe, non seulement parce que les Latinos sont beaucoup plus nombreux (17, 8\% de la population nationale en 2017 contre 9,0\% en 1990) et d'origines nationales très diverses, mais aussi parce qu'ils sont beaucoup plus dispersés sur le territoire étatsunien. Leur présence devient visible dans les régions où historiquement, avant les années 1990, ils étaient très peu nombreux et attiraient peu l'attention.

Depuis le début des années 2000, des sociologues, socio-économistes, et ethnographes partent à la découverte de ces populations nouvelles ${ }^{1}$. La particularité de ce recueil est de rendre compte de la population latino de la région Midwest des Etats-Unis, qui consiste en 12 Etats - Illinois, Indiana, Iowa, Kansas, Michigan, Minnesota, Missouri, Nebraska, North Dakota, Ohio, South Dakota, Wisconsin - où habite environ 20\% de la 
population nationale, soit 70 millions d'habitants environ. En 2010 les Latinos ne constituaient que $7,6 \%$ de la population de la région, mais ce chiffre représentait un taux de croissance spectaculaire de $73 \%$ depuis 2000 .

Parmi les auteurs des 17 chapitres se trouvent des professeurs de Latino Studies, American Studies, histoire, langue espagnole, lettres, Performance Studies, sciences de l'éducation, communication, histoire de l'art. Les approches de la vie sociale et culturelle des Latinos ne sont pas celles des sociologues ou des socio-démographes mais relèvent davantage de l'histoire contemporaine et de l'ethnographie.

Si les Mexicains ont toujours été majoritaires, on trouve des traces dans le Midwest de Latinos de diverses origines. Dans leur introduction les trois co-éditeurs définissent le Midwest comme « une région de latinidades à la fois distinctes et enchevêtrées, rurales et urbaines, établies et émergentes, qui se sont forgées depuis un siècle et demi par les migrations du travail, l'urbanisation, la production de lieux (placemaking) et la production culturelle. Les latinidades examinées sont à la fois transnationales, transrégionales, intra-régionales et virtuelles... » (p. 18).

Dans ce livre qui se focalise principalement sur l'époque contemporaine, plusieurs auteurs s'attachent à rappeler un passé encore méconnu. Bien avant l'arrivée des Mexicains recrutés dans la classe ouvrière à Chicago à partir de 1919 (voir le chapitre de Michael Innis-Jiménez), des ouvriers agricoles et des cowboys (vaqueros) mexicains avaient laissé des traces dans de nombreuses zones rurales du Midwest. A titre d'illustration, José E. Limón, spécialiste de la poésie et des chansons des Mexicains du Sud-Ouest, livre dans son chapitre les touchantes paroles d'une ballade intitulée "El corrido de Kiansis", composée par des vaqueros mexicains itinérants dans les années 1860 après avoir été témoins dans le Kansas de la mort d'un membre de leur équipe.

6 Le livre se compose de cinq parties thématiques. Le titre de la première partie - The Browning of the Midwest - se réfère à des processus locaux d'implantation qui, en modifiant le paysage social et culturel, pose un défi permanent aux Latinos perçus comme très différents (« racialisés »). D'où leurs efforts pour défendre leurs droits et obtenir la reconnaissance de la société majoritaire. La chronique contemporaine par Aidé Acosta de la vie des immigrés et leurs pratiques culturelles dans la petite ville de Lorraine (Illinois) en zone rurale, est particulièrement éloquente de ce point de vue.

7 La deuxième partie, "Essential Laborers and Neighbors", porte sur les travailleurs immigrés, les flux migratoires qui les ont amenés dans la région, l'histoire de leurs associations, leurs luttes syndicales, enfin leur transformation, à terme, en en « voisins ». Cette partie inclut une brève histoire des ouvriers mexicains des quartiers sud de Chicago à partir de 1919 ; une évocation de la rencontre, et des rapports forts complexes, entre Mexicains et Portoricains à Chicago après 1945 ; enfin une étude contemporaine de Perry, ville industrielle de 7000 habitants dans l'Iowa central où la population latino est passée de zéro avant 1990 à $35 \%$ en 2010. Il ressort de ce chapitre que les enfants des salariés de l'usine locale sont nombreux à résister au stéréotype qui fait de tous les Latinos des travailleurs manuels.

8 L'école et les questions linguistiques fournissent la matière de la troisième partie. A signaler dans cette partie, une étude très complète des pratiques linguistiques des Latinos et des pratiques de l'enseignement des langues dans le Midwest dans une perspective nationale (Kim Potowski) ; une étude réalisée dans une zone rurale du sudouest de l'Iowa, près d'une usine de conditionnement de la viande, sur les difficultés des rapports entre les parents immigrés et les enseignants de l'école locale ; enfin la 
chronique de la lutte, en 2013, pour sauver un programme de Latino Studies menacé de coupes budgétaires et d'extinction à l'Université du Nebraska.

9 «Performeando the Midwest »-- beau néologisme spanglish - est le titre de la quatrième partie, qui a pour thème sur les arts du spectacle. Cette partie débute sur l'histoire d'une œuvre filmée de l'artiste Ana Mendieta (1948-1985) dans l'Iowa, où elle a vécu une partie de son enfance, juste après la révolution cubaine ; une chronique du spectacle gay et transsexuel contemporain à Chicago accompagnée d'une réflexion sur l'esthétique queer; enfin l'histoire et pré-histoire de la fondation en 2009 du musée des Latinos de Detroit, connu sous le nom du Museo del Norte (Musée du Nord).

La dernière partie ("Movimientos») aborde les mouvements sociaux auxquels ont participé des Latinos du Midwest : mouvements religieux, politiques, syndicaux et/ou de défense des minorités. Cette partie commence avec l'histoire des Mennonites, courant religieux d'origine suisse-allemande qui a fait des adeptes parmi des Mexicains du Midwest à partir des années 1930 ; ensuite, une évocation trop courte (3 pages!) des Young Lords, organisation militante portoricaine fondée en 1969 à Chicago ; une chronique du travail de solidarité active entrepris par des Mexicains de l'Iowa, dès 1965, avec le syndicat des United Farm Workers de César Chávez en Californie ; et une histoire, sur la base de témoignages oraux, de plusieurs femmes latinas de l'Iowa et du Wisconsin ayant joué des rôles de leaders de différentes sortes, depuis les années 1940. La partie se termine sur une chronique contemporaine des formes novatrices de lutte des jeunes sans-papiers à Chicago, centre névralgique avec Los Angeles du mouvement des droits des immigrés à l'échelle nationale.

11 La postface de Frances R. Aparicio constitue un véritable chapitre à part. Sur la base d'entretiens avec des jeunes de Chicago d'origine mixte «interlatina/o » - le mélange "MexiRican» (mexicain et portoricain) étant le cas de figure le plus fréquent - elle réfléchit sur l'irréductible pluralité des "significations sociales et intimes» de la latinidad, d'où les différences de langue, d'origine nationale et de classe sociale ne disparaissent pas comme par magie (p. 283).

Puisque ce recueil vise en effet à rendre compte des Latinos du Midwest dans toute la diversité des populations, des lieux et des situations, son effet " patchwork » n'est pas nécessairement un mal. Si l'on peut regretter l'absence de quelques tableaux statistiques pour préciser davantage les données démographiques, c'est un livre de référence utile qui comporte une bibliographie très complète (p. 293-316) et fournira beaucoup d'idées aux chercheurs.

\section{NOTES}

1. Pour ne citer que deux titres : Douglas S. Massey (éd.), New Faces in New Places: The Changing Geography of American Immigration, Russell Sage Foundation, 2008 ; et, pour la région du Midwest, Ann Millard et Jorge Chapa (éds.), Apple Pie and Enchiladas: Latino Newcomers in the Rural Midwest, University of Texas Press, 2004. La bibliographie du Latina/o Midwest Reader (p. 293-316) est très complète. 


\section{AUTEURS}

\section{JAMES COHEN}

James Cohen est professeur à l'Université de Paris 3 Sorbonne Nouvelle, CREW (EA 4399), auteur de À la poursuite des « illégaux ». Politiques et mouvements anti-immigrés aux États-Unis, Éditions Le Croquant, 2012. 